\title{
Génotypes au locus de sensibilité à l'halothane et caractères de croissance et de carcasse dans une F2 Piétrain x Large White
}

\author{
R Hanset, C Dasnois, S Scalais, C Michaux, L Grobet \\ Service de génétique factorielle et moléculaire, faculté de médecine vétérinaire \\ de l'université de Liège, 20, bd de Colonster, B 43, B-4000 Liège, Belgique
}

(Reçu le 27 octobre 1993; accepté le 28 septembre 1994)

\begin{abstract}
Résumé - Une F2 de 1500 porcs environ a été produite à partir d'un croisement Piétrain $\times$ Large White. Le croisement a été construit de manière à permettre l'identification des 3 génotypes $(N N, N n$ et $n n)$ au locus $\mathrm{Hal}$ de sensibilité à l'halothane. On a estimé les effets de ces 3 génotypes sur une série de critères zootechniques (croissance, conformation, composition de carcasse, qualité de viande) ainsi que les contributions de ce locus et du sexe à la variation totale de ces différents caractères. L'effet du gène (écart entre homozygotes) et l'écart de dominance ont aussi été calculés. Les effets les plus marqués du gène concernent surtout les critères influençant la conformation (pourcentage de morceaux nobles et longueur de la carcasse) et le pH1 du muscle long dorsal. Leur amplitude s'élève approximativement à un écart type. L'expression chez l'hétérozygote de ces mêmes critères de conformation explique l'avantage sélectif dont a bénéficié le gène, même en dose simple, lors de la formation de la race. Comme le sujet de la F2 homozygote $n n$ ne peut être confondu avec un Piétrain pur, en ce qui concerne la composition de la carcasse et la conformation, on en conclut que, si le génotype $n n$ est une condition nécessaire pour constituer l'entité biologique qu'est le Piétrain, cette condition n'est toutefois pas suffisante et que, dès lors, d'autres gènes dont l'action globale sur la composition de la carcasse est analogue à celle de l'allèle $n$ participent aussi au déterminisme de l'entité Piétrain.
\end{abstract}

croisement Piétrain $\times$ Large White / locus Hal / croissance / carcasse

Summary - Genotypes at the locus for halothane sensitivity and performance in a Piétrain $\times$ Large White F2. An F2 consisting of about 1500 pigs was produced from a Piétrain $\times$ Large White cross. The experiment was designed so as to allow the identification of the 3 genotypes (NN, Nn and $\mathrm{nn}$ ) at the locus for halothane sensitivity. The effects of these 3 genotypes on production traits were estimated as well as the contributions of this locus and sex to their total variation. The criteria considered were: growth; conformation indices carcass composition; and meat quality. The gene effect (difference between homozygotes) and the dominance deviation were also calculated. The most pronounced effects of 
this locus concern mainly those criteria related to conformation, such as lean percentage or carcass length, and the $p H$ measurement (eye muscle). Their magnitude is approximately 1 standard deviation. The partial expression in the heterozygote of the effects on conformation explains why even a single copy of the gene was favoured by selection during the course of breed formation. Moreover, as an F2 individual of $\mathrm{nn}$ genotype cannot be mistaken for a pure Piétrain regarding carcass composition and conformation, it is concluded that the nn genotype is a necessary but not sufficient condition to make a Piétrain, other genes being involved whose action on carcass composition is similar to that of the $n$ allele.

Piétrain / Large White / cross / halothane locus / production trait

\section{INTRODUCTION}

L'hypertrophie musculaire et la sensibilité à l'halothane sont 2 caractéristiques bien connues du porc Piétrain. Dans cette race où la conformation est un critère essentiel de sélection, tout gène favorable au développement musculaire voit sa fréquence s'élever et tend vers la fixation. Ce fut le cas de l'allèle $n$ au locus $H a l$ de la sensibilité à l'halothane dont on connaît l'effet majeur sur le développement musculaire et sur la conformation (Ollivier et al, 1975; Eikelenboom et al, 1978; Webb et Jordan, 1978; Monin et al, 1980; Lampo, 1981). Une expérience a été entreprise dans le but d'évaluer la part du locus $\mathrm{Hal}$ dans le déterminisme de l'entité biologique qu'est le porc Piétrain. Dans cet article, on étudie les effets des différents génotypes à ce locus sur les caractères de croissance de conformation, de carcasse et de qualité de viande au sein d'un F2 produite à partir d'un croisement Piétrain $\times$ Large White.

\section{MATÉRIEL ET MÉTHODES}

L'expérience a débuté par le choix en Belgique de verrats Piétrain et en France (INRA) de truies Large White homozygotes respectivement pour les allèles $B$ et $A$ au locus de la $P H I$ (phosphohexose isomérase). Ces verrats et ces truies étaient également, avec une probabilité proche de la certitude, homozygotes respectivement pour les allèles $n$ et $N$ au locus $\mathrm{Hal}$. Les résultats des croisements ultérieurs, tous les sujets de la F1 étant $\mathrm{Hal}^{-}$de même que 3/4 des sujets de la F2, devaient conforter cette supposition. Les deux loci concernés $P H I$ et $H a l$ sont étroitement liés (Jørgensen et al, 1976). On sait que, en outre, ces 2 races sont respectivement homozygotes $i i$ et $I I$, gène de coloration indépendant des précédents, le Piétrain présentant le tacheté noir récessif et le Large White le blanc dominant. Le croisement de ces 2 races devait dès lors donner naissance à une $\mathrm{F} 1$ de génotype $\frac{B n}{A N} \frac{i}{I}$, tous sujets halothane négatifs, $A B$ pour la $P H I$ et de robe blanche. La F2 dérive donc du croisement $\frac{B n}{A N} \frac{i}{I} \times \frac{B n}{A N} \frac{i}{I}$. Les parents F1 se composaient de 81 truies et 32 verrats. Dans cette F2, on attend que, en dehors des recombinants, les sujets $B B$ soient homozygotes $n n$ et $\mathrm{Hal}^{+}$, les sujets $\mathrm{AB}$ hétérozygotes $\mathrm{Nn}$ et $\mathrm{Hal}^{-}$, les sujets $A A$ homozygotes $N N$ et $\mathrm{Hal}^{-}$. 
Si le typage pour le marqueur biochimique est associé au test à l'halothane, on peut ainsi reconnaître les recombinants $A B n n, B B N n, A A n n, B B N N$. Dès lors, en procédant de la sorte, le génotype au locus $H a l$ pouvait être déterminé avec une très grande précision.

Le test à l'halothane a été réalisé à l'âge moyen de $60 \mathrm{j}$ et au poids moyen de $18 \mathrm{~kg}$. La durée d'exposition à un mélange gazeux contenant $5 \%$ d'halothane était de $5 \mathrm{~min}$. Le typage pour la $P H I$ a été fait selon la méthode décrite par Widar et al (1975). Le sevrage des porcelets intervient à l'âge de $35 \mathrm{j}$, moment de la castration des mâles. L'engraissement proprement dit débute après une période de post-sevrage d'une durée de 4 sem. Les animaux sont élevés par groupe de 10, choisis en fonction de l'âge, et reçoivent une alimentation ad libitum. La composition de l'aliment pour le porcelet est la suivante : protéine brute $18 \%$, matières grasses brutes $4 \%$, cellulose brute $3,5 \%$, cendres brutes $6 \%$, lysine $1,15 \%$, additifs : vit $\mathrm{A}, \mathrm{D} 3, \mathrm{E}$, sulfate cuivrique, facteur de croissance : olaquindox. L'aliment pour l'engraissement contient : protéine brute $17,5 \%$, matières grasses brutes $3,0 \%$, cellulose brute $5,5 \%$, cendres brutes $6,0 \%$, additifs : vit $\mathrm{A}, \mathrm{D} 3, \mathrm{E}$, sulfate cuivrique, facteur de croissance : avoporcine.

Les critères zootechniques sont définis comme suit :

Gain quotidien : entre le poids initial (20 kg environ) et le poids final (105 kg environ). Le poids vif final est pris la veille de l'abattage, avant la mise à jeun. La durée du jeûne est d'environ $24 \mathrm{~h}$.

Notes de conformation (au sevrage et à la fin de l'engraissement). Cinq régions sont prise en compte : l'épaule (d'effacée à saillante), la poitrine (d'ovoïde à cylindrique), le dos (de rond avec carré non saillant à large carré en saillie, gouttière médiane), la croupe (de l'absence de creux au-dessus de la queue à la présence d'un creux au-dessus de la queue), le jambon (d'allongé sans sillons à globuleux avec sillons intermusculaires).

Pour chacune de ces régions, l'échelle de pointage comporte les notes 0,1 et 2 . Ces notes individuelles sont additionnées. La note maximum est 10 (porc culard typique), la note minimum est 0 (porc type Large White).

Critères de carcasse : mesurés sur la demi-carcasse gauche.

La longueur représente la distance entre la première côte et la pointe du pubis. L'épaisseur du gras dorsal est mesurée i) au niveau du cou, ii) au niveau de la première vertèbre lombaire, iii) au niveau de la croupe. L'épaisseur moyenne de gras dorsal est la moyenne des 3 mesures ci-dessus. Le rendement à l'abattage est donné par le poids de la carcasse ( 2 fois le poids de la demi-carcasse gauche, pesée le lendemain de l'abattage) divisé par le poids vif final. La découpe est pratiquée sur la demi-carcasse gauche, le lendemain de l'abattage.

Les morceaux de la découpe envisagés sont : épaule, carré, jambon, bardière, paroi ventrale (découpe dite Bruxelloise illustrée dans Hanset et Van Snick (1972)). La proportion de morceaux maigres est égale à la somme des pourcentages de l'épaule, du carré, du jambon tandis que la proportion des morceaux gras concerne outre la bardière et la paroi ventrale, le collier et le saindoux (déchets gras). Deux indices de compacité sont calculés : indice de compacité 1 : il est obtenu en divisant le poids de la carcasse par sa longueur ; indice de compacité 2 : il s'obtient en divisant par la longueur de la carcasse le poids des morceaux maigres (demi-carcasse) multiplié par 2. Les mesures du $\mathrm{pH}$ sont effectuées $1 \mathrm{~h}$ et $24 \mathrm{~h}$ après l'abattage 
au niveau du carré (muscle Longissimus dorsi) (première vertèbre lombaire) et au niveau du jambon (muscle Gracilis). Quant aux classes commerciales, leur description a été donnée par Kintaba et al (1981). Par ordre décroissant, on a les classes EE, E, AA, A1, A2, A3.

Le modèle d'analyse retenu comporte les sources de variation suivantes :

i) le génotype au locus $\mathrm{Hal}$ ( 3 niveaux);

ii) le sexe ( 2 niveaux);

iii) la robe ( 2 niveaux : blanc ou pie-noire, correspondant à la ségrégation des 2 allèles de couleur $I$ et $i)$;

iv) année-mois d'abattage (42 périodes s'échelonnant de janvier 1986 à septembre 1989).

En outre, le poids d'abattage figure dans le modèle comme covariable. Le nombre de porcs analysés varie de 1448 à 1632 selon le critère envisagé.

Le nombre d'animaux de génotype $N N$ est compris entre 409 et 427 selon le caractère. Pour le génotype $N n$, ce nombre varie de 812 à 840 et pour le génotype $n n$, de 353 à 365 . Ces fréquences concernant des porcs ayant atteint le terme de l'engraissement, elles n'ont pas valeur de données de ségrégation. Le nombre de castrats est compris entre 793 et 817 et le nombre de femelles entre 781 et 815 .

Une analyse préalable comportant l'interaction génotype $\times$ sexe avait révélé une participation négligeable de ce terme supplémentaire. Comme le nombre de porcs abattus chaque semaine était très variable, il a été jugé préférable de réaliser un groupement mensuel des données. D'ailleurs, il est apparu que, dans nos conditions d'abattage, la substitution dans le modèle du jour d'abattage au mois d'abattage n'avait pas d'effet sensible sur les résultats concernant les critères de qualité de viande.

En outre, les contributions (en \%) du locus et du sexe à cette variation ont été calculées en divisant les sommes de carrés correspondant à ces effets par la somme totale des carrés. D'autre part, l'effet du gène (écart entre les homozygotes $n n$ et $N N$ ) au locus $H a l-2 a-a$ été estimé par la différence entre la valeur métrique du génotype $n n$ et celle du génotype $N N$ tandis que l'écart de dominance - d l'a été par l'écart entre la valeur métrique de l'hétérozygote et la valeur métrique moyenne des 2 homozygotes. En outre, l'effet du gène a été standardisé en le divisant par l'écart type résiduel tandis que l'écart de dominance a été exprimé sous forme relative en le divisant par la demi-différence entre les 2 homozygotes. La signification statistique des effets $2 \mathrm{a}$ et $\mathrm{d}$ a été testée à l'aide des contrastes respectifs $10-1$ et $1-21$ appliqués aux 3 génotypes au locus $\mathrm{Hal}$. Les analyses statistiques ont été réalisées avec la procédure GLM de SAS (1985).

\section{RÉSULTATS}

Les moyennes des moindres carrés correspondant aux 3 génotypes au locus $H a l$ et aux 2 sexes sont données au tableau I. L'effet du locus $\mathrm{Hal}$ est significatif pour tous les caractères envisagés bien que sa contribution à la variation diffère fortement d'un caractère à l'autre. Les 3 génotypes de la F2 sont encore comparés entre eux et avec les races Large White et Piétrain dans une série de figures : pour le gain journalier (fig 1), la longueur de carcasse (fig 2), le pourcentage de morceaux 
Tableau I. Moyennes des moindres carrés ( \pm erreur standard) correspondant aux effets «génotype» et «sexe» du modèle.

\begin{tabular}{|c|c|c|c|c|c|c|c|}
\hline \multirow[b]{2}{*}{ Caractères } & \multicolumn{3}{|c|}{ Génotype Halothane } & \multicolumn{4}{|c|}{ Sexe } \\
\hline & $\mathrm{NN}$ & $\mathrm{Nn}$ & $\mathrm{nn}$ & $\begin{array}{c}\mathrm{R}^{2} \\
\mathrm{P}\end{array}$ & Castrats & Femelles & $\begin{array}{l}\mathrm{R}^{2} \\
\mathrm{P}\end{array}$ \\
\hline Gain quotidien (kg) & $\begin{array}{r}0,706 \\
\pm 0,005\end{array}$ & $\begin{array}{r}0,731 \\
\pm 0,004\end{array}$ & $\begin{array}{r}0,718 \\
\pm 0,005\end{array}$ & $\begin{array}{l}0,90 \\
* * *\end{array}$ & $\begin{array}{r}0,742 \\
\pm 0,004\end{array}$ & $\begin{array}{r}0,694 \\
\pm 0,004\end{array}$ & $\begin{array}{l}4,80 \\
* * *\end{array}$ \\
\hline Longueur $(\mathrm{cm})$ & $\begin{array}{r}80,93 \\
\pm 0,12\end{array}$ & $\begin{array}{r}80,48 \\
\pm 0,09\end{array}$ & $\begin{array}{r}79,03 \\
\pm 0,13\end{array}$ & $\begin{array}{l}7,18 \\
* * *\end{array}$ & $\begin{array}{r}79,53 \\
\pm 0,10\end{array}$ & $\begin{array}{r}80,76 \\
\pm 0,10\end{array}$ & $\begin{array}{l}5,65 \\
* * *\end{array}$ \\
\hline $\begin{array}{l}\text { Épaisseur du gras } \\
\text { dorsal }(\mathrm{cou})(\mathrm{cm})\end{array}$ & $\begin{array}{r}3,75 \\
\pm 0,03\end{array}$ & $\begin{array}{r}3,73 \\
\pm 0,02\end{array}$ & $\begin{array}{r}3,59 \\
\pm 0,03\end{array}$ & $\begin{array}{l}0,90 \\
* * *\end{array}$ & $\begin{array}{r}3,85 \\
\pm 0,02\end{array}$ & $\begin{array}{r}3,54 \\
\pm 0,02\end{array}$ & $\begin{array}{l}5,60 \\
* * *\end{array}$ \\
\hline $\begin{array}{l}\text { Épaisseur du gras dorsal } \\
\left(1^{\text {re }} \text { vertèbre }\right. \\
\text { lombaire })(\mathrm{cm})\end{array}$ & $\begin{array}{r}2,59 \\
\pm 0,03\end{array}$ & $\begin{array}{r}2,59 \\
\pm 0,02\end{array}$ & $\begin{array}{r}2,51 \\
\pm 0,03\end{array}$ & $\begin{array}{c}0,33 \\
*\end{array}$ & $\begin{array}{r}2,74 \\
\pm 0,02\end{array}$ & $\begin{array}{r}2,39 \\
\pm 0,02\end{array}$ & $\begin{array}{l}9,56 \\
* * *\end{array}$ \\
\hline $\begin{array}{l}\text { Épaisseur du gras dorsal } \\
\text { (croupe) }(\mathrm{cm})\end{array}$ & $\begin{array}{r}1,70 \\
\pm 0,02\end{array}$ & $\begin{array}{r}1,66 \\
\pm 0,02\end{array}$ & $\begin{array}{r}1,42 \\
\pm 0,03\end{array}$ & $\begin{array}{l}4,12 \\
* * *\end{array}$ & $\begin{array}{r}1,77 \\
\pm 0,02\end{array}$ & $\begin{array}{r}1,42 \\
\pm 0,02\end{array}$ & $\begin{array}{l}11,37 \\
* * *\end{array}$ \\
\hline $\begin{array}{l}\text { Épaisseur moyenne du } \\
\text { gras dorsal }(\mathrm{cm})\end{array}$ & $\begin{array}{r}2,68 \\
\pm 0,02\end{array}$ & $\begin{array}{r}2,66 \\
\pm 0,02\end{array}$ & $\begin{array}{r}2,51 \\
\pm 0,02\end{array}$ & $\begin{array}{l}2,08 \\
* * *\end{array}$ & $\begin{array}{r}2,78 \\
\pm 0,02\end{array}$ & $\begin{array}{r}2,45 \\
\pm 0,02\end{array}$ & $\begin{array}{l}13,38 \\
* * *\end{array}$ \\
\hline Rendement (\%) & $\begin{array}{r}80,15 \\
\pm 0,10\end{array}$ & $\begin{array}{r}80,12 \\
\pm 0,07\end{array}$ & $\begin{array}{r}81,03 \\
\pm 0,10\end{array}$ & $\begin{array}{l}3,72 \\
* * *\end{array}$ & $\begin{array}{r}80,39 \\
0,07\end{array}$ & $\begin{array}{r}80,48 \\
0,07\end{array}$ & $\begin{array}{l}0,05 \\
\text { NS }\end{array}$ \\
\hline Carré (\%) & $\begin{array}{r}24,08 \\
\pm 0,07\end{array}$ & $\begin{array}{r}24,38 \\
\pm 0,05\end{array}$ & $\begin{array}{r}25,05 \\
\pm 0,08\end{array}$ & $\begin{array}{l}4,25 \\
* * *\end{array}$ & $\begin{array}{r}23,76 \\
\pm 0,06\end{array}$ & $\begin{array}{r}25,25 \\
\pm 0,06\end{array}$ & $\begin{array}{l}19,51 \\
* * *\end{array}$ \\
\hline Jambon (\%) & $\begin{array}{r}22,78 \\
\pm 0,06\end{array}$ & $\begin{array}{r}23,12 \\
\pm 0,05\end{array}$ & $\begin{array}{r}23,72 \\
\pm 0,06\end{array}$ & $\begin{array}{l}6,15 \\
* * *\end{array}$ & $\begin{array}{r}22,81 \\
\pm 0,05\end{array}$ & $\begin{array}{r}23,60 \\
\pm 0,05\end{array}$ & $\begin{array}{l}8,70 \\
* * *\end{array}$ \\
\hline Épaule (\%) & $\begin{array}{r}16,31 \\
\pm 0,04\end{array}$ & $\begin{array}{r}16,45 \\
\pm 0,03\end{array}$ & $\begin{array}{r}16,77 \\
\pm 0,04\end{array}$ & $\begin{array}{l}4,96 \\
* * *\end{array}$ & $\begin{array}{r} \pm 16,46 \\
0,03\end{array}$ & $\begin{array}{r}16,56 \\
\pm 0,03\end{array}$ & $\begin{array}{l}0,43 \\
* *\end{array}$ \\
\hline $\begin{array}{l}\text { Morceaux } \\
\text { maigres (\%) }\end{array}$ & $\begin{array}{r}63,17 \\
\pm 0,13\end{array}$ & $\begin{array}{r}63,95 \\
\pm 0,10\end{array}$ & $\begin{array}{r}65,54 \\
\pm 0,14\end{array}$ & $\begin{array}{l}7,68 \\
* * *\end{array}$ & $\begin{array}{r}63,04 \\
\pm 0,10\end{array}$ & $\begin{array}{r}65,40 \\
\pm 0,10\end{array}$ & $\begin{array}{l}15,23 \\
* * *\end{array}$ \\
\hline Bardière (\%) & $\begin{array}{r}7,32 \\
\pm 0,07\end{array}$ & $\begin{array}{r}7,21 \\
\pm 0,05\end{array}$ & $\begin{array}{r}6,68 \\
\pm 0,07\end{array}$ & $\begin{array}{l}2,24 \\
* * *\end{array}$ & $\begin{array}{r}7,81 \\
\pm 0,05\end{array}$ & $\begin{array}{r}6,33 \\
\pm 0,05\end{array}$ & $\begin{array}{l}20,85 \\
* * *\end{array}$ \\
\hline $\begin{array}{l}\text { Paroi ventrale } \\
(\%)\end{array}$ & $\begin{array}{r}16,34 \\
\pm 0,05\end{array}$ & $\begin{array}{r}16,07 \\
\pm 0,04\end{array}$ & $\begin{array}{r}15,75 \\
\pm 0,06\end{array}$ & $\begin{array}{l}3,31 \\
* * *\end{array}$ & $\begin{array}{r}16,33 \\
\pm 0,04\end{array}$ & $\begin{array}{r}15,78 \\
\pm 0,04\end{array}$ & $\begin{array}{l}5,87 \\
* * *\end{array}$ \\
\hline $\begin{array}{l}\text { Morceaux } \\
\text { gras (\%) }\end{array}$ & $\begin{array}{r}30,04 \\
\pm 0,13\end{array}$ & $\begin{array}{r}29,27 \\
\pm 0,10\end{array}$ & $\begin{array}{r}27,85 \\
\pm 0,13\end{array}$ & $\begin{array}{l}6,58 \\
* * *\end{array}$ & $\begin{array}{r}30,39 \\
\pm 0,10\end{array}$ & $\begin{array}{r}27,72 \\
\pm 0,10\end{array}$ & $\begin{array}{l}19,77 \\
* * *\end{array}$ \\
\hline $\begin{array}{l}\text { Conformation } \\
\quad \text { au sevrage ( } 0 \text { à } 10)\end{array}$ & $\begin{array}{r}4,47 \\
\pm 0,12\end{array}$ & $\begin{array}{r}5,08 \\
\pm 0,09\end{array}$ & $\begin{array}{r}6,40 \\
\pm 0,13\end{array}$ & $\begin{array}{l}8,94 \\
* * *\end{array}$ & $\begin{array}{r}5,48 \\
\pm 0,09\end{array}$ & $\begin{array}{r}5,15 \\
\pm 0,09\end{array}$ & $\begin{array}{l}0,53 \\
* *\end{array}$ \\
\hline $\begin{array}{l}\text { Conformation } \\
\text { finale (0 à 10) }\end{array}$ & $\begin{array}{r}4,84 \\
\pm 0,10\end{array}$ & $\begin{array}{r}5,47 \\
\pm 0,08\end{array}$ & $\begin{array}{r}6,71 \\
\pm 0,11\end{array}$ & $\underset{* * *}{12,00}$ & $\begin{array}{r}5,74 \\
\pm 0,09\end{array}$ & $\begin{array}{r}5,60 \\
\pm 0,09\end{array}$ & $\begin{array}{l}0,15 \\
+\end{array}$ \\
\hline $\begin{array}{l}\text { Indice } \\
\text { de compacité } 1\end{array}$ & $\begin{array}{r}1,025 \\
\pm 0,002\end{array}$ & $\begin{array}{r}1,029 \\
\pm 0,002\end{array}$ & $\begin{array}{r}1,059 \\
\pm 0,002\end{array}$ & $\begin{array}{l}6,62 \\
* * *\end{array}$ & $\begin{array}{r}1,044 \\
\pm 0,002\end{array}$ & $\begin{array}{r}1,030 \\
\pm 0,002\end{array}$ & $\begin{array}{l}1,76 \\
* * *\end{array}$ \\
\hline $\begin{array}{l}\text { Indice } \\
\quad \text { de compacité } 2\end{array}$ & $\begin{array}{r}0,623 \\
\pm 0,002\end{array}$ & $\begin{array}{r}0,635 \\
+0,001\end{array}$ & $\begin{array}{r}0,669 \\
\pm 0,002\end{array}$ & $\begin{array}{c}16,34 \\
* * *\end{array}$ & $\begin{array}{r}0,634 \\
\pm 0,001\end{array}$ & $\begin{array}{r}0,650 \\
\pm 0,001\end{array}$ & $\begin{array}{l}3,90 \\
* * *\end{array}$ \\
\hline
\end{tabular}


Tableau I (suite)

\begin{tabular}{lrrrrrrr}
\hline & \multicolumn{3}{c}{ Génotype Halothane } & \multicolumn{3}{c}{ Sexe } \\
Caractères & $\mathrm{NN}$ & $\mathrm{Nn}$ & $\mathrm{nn}$ & $\mathrm{R}^{2}$ & Castrats & Femelles & $\mathrm{R}^{2}$ \\
& & & & $\mathrm{P}$ & & & $\mathrm{P}$ \\
\hline pH1 carré & 6,15 & 5,91 & 5,80 & 11,25 & 5,97 & 5,94 & 0,11 \\
$\quad(\mathrm{~m}$ Longissimus dorsi) & $\pm 0,02$ & $\pm 0,01$ & $\pm 0,02$ & $* * *$ & $\pm 0,01$ & $\pm 0,01$ & $\mathrm{NS}$ \\
pH1 jambon & 6,22 & 6,19 & 6,15 & 0,62 & 6,22 & 6,16 & 0,85 \\
$\quad(\mathrm{~m}$ Gracilis $)$ & $\pm 0,02$ & $\pm 0,01$ & $\pm 0,02$ & $* *$ & $\pm 0,01$ & $\pm 0,01$ & $* * *$ \\
pH24 carré & 6,01 & 5,96 & 5,95 & 0,77 & 5,99 & 5,95 & 0,63 \\
$\quad(\mathrm{~m}$ Longissimus dorsi) & $\pm 0,01$ & $\pm 0,01$ & $\pm 0,01$ & $* * *$ & $\pm 0,01$ & $\pm 0,01$ & $* * *$ \\
pH24 jambon & 6,25 & 6,26 & 6,30 & 0,38 & 6,32 & 6,22 & 2,57 \\
$\quad$ (m Gracilis) & $\pm 0,01$ & $\pm 0,01$ & $\pm 0,01$ & $*$ & & & $* * *$ \\
\hline
\end{tabular}

$\left[R^{2}:\right.$ part de la variation due à l'effet considéré. NS : $P>0,10 ;+: P<0,10$; ${ }^{*}: P<0,05$; $^{* *}: P<0,01$; $^{* * *}: P<0,001$.

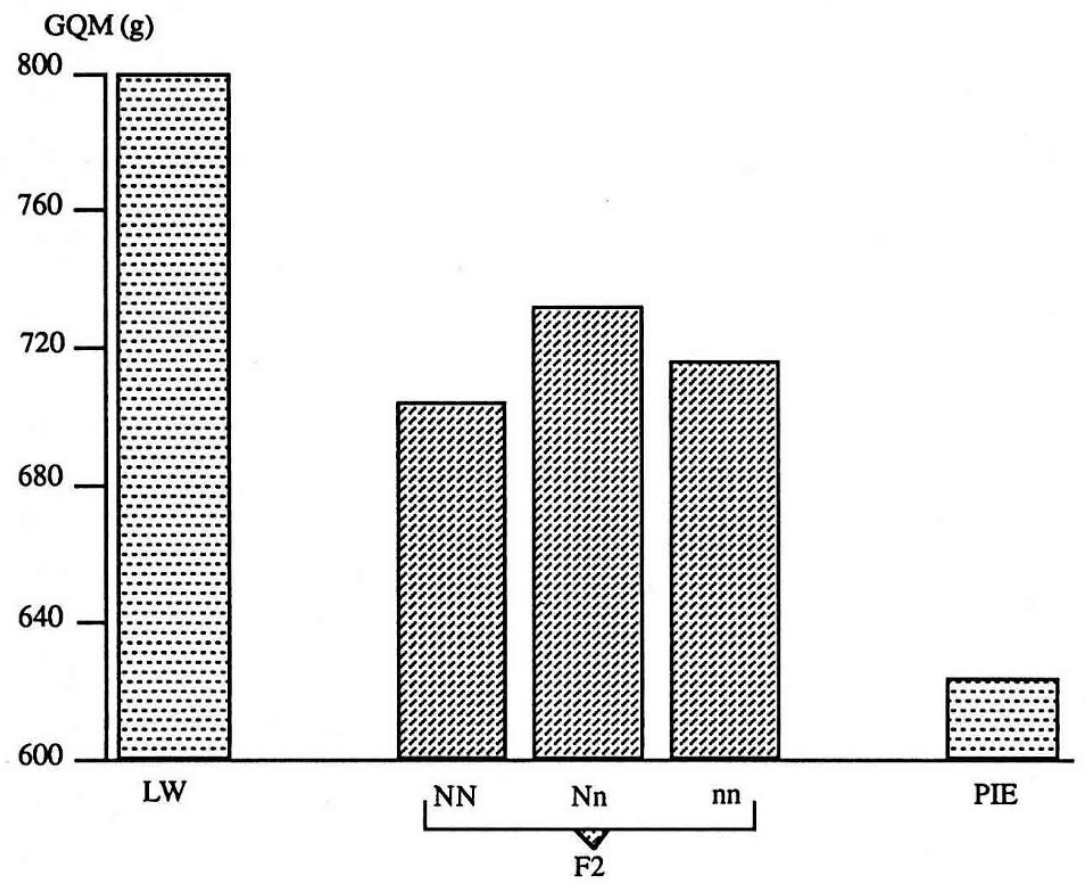

Fig 1. Gain quotidien des 3 génotypes de la F2 et des races Large White (LW) et Piétrain (PIE). 


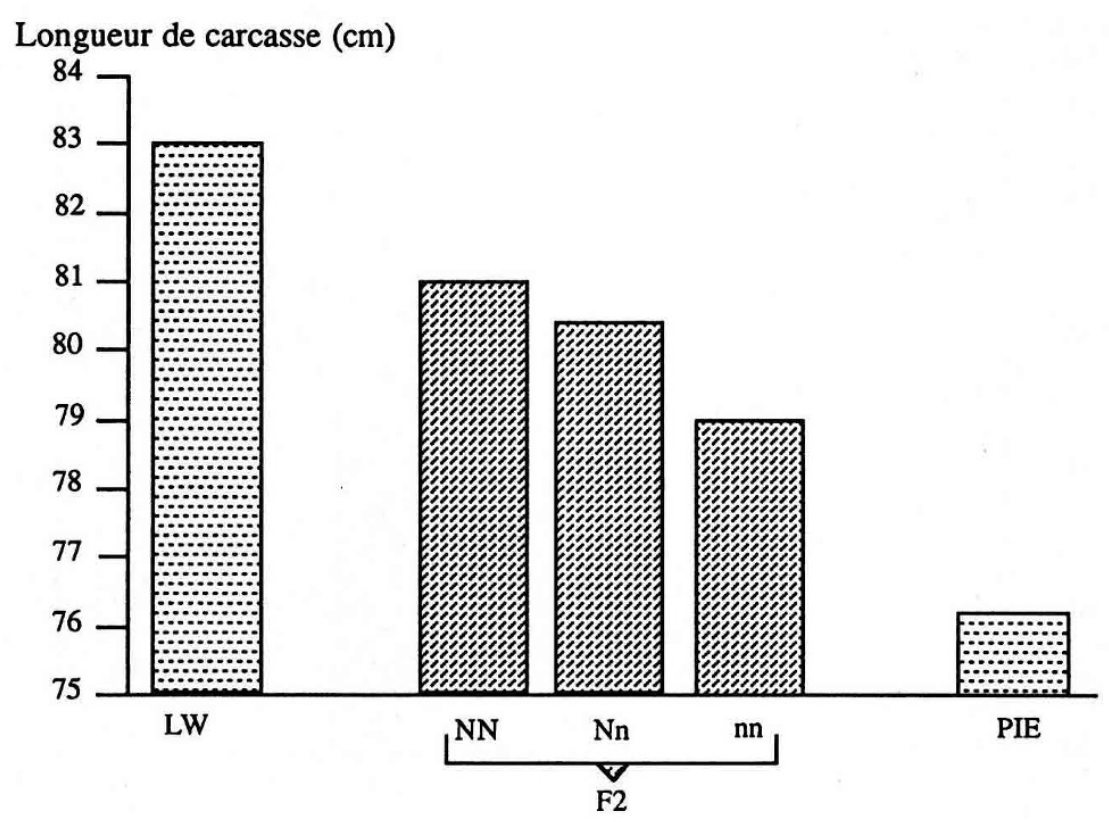

Fig 2. Longueur de carcasse des 3 génotypes de la F2 et des races Large White (LW) et Piétrain (PIE).

maigres (fig 3), la mesure du pH1 du carré (fig 4). Les moyennes raciales utilisées pour cette comparaison ont été calculées sur des animaux élevés antérieurement dans les mêmes conditions que les sujets de l'expérience et en veillant à l'équilibre des sexes (effectifs : LW $n=34$; Pié $n=270$ ). Toutefois, seule une valeur indicative leur sera accordée.

En passant du génotype $N N$ au génotype $n n$, on observe une réduction de la longueur de la carcasse $(-1,9 \mathrm{~cm})$, de l'épaisseur du gras dorsal (effet moins prononcé pour l'épaisseur 1 et surtout pour l'épaisseur 2), du pourcentage de morceaux gras $(-2,18 \%)$, des $\mathrm{pH} 1$ du carré et du jambon, du pH24 du carré, une augmentation du rendement à l'abattage $(+0,9 \%)$ de la proportion de morceaux maigres $(+2,4 \%)$, des notes de conformation et des indices de compacité. L'indice de compacité 2 en combinant le pourcentage de morceaux maigres et la longueur de la carcasse a un pouvoir discriminant plus prononcé que l'indice de compacité 1 .

Seul résultat inattendu, le pH24 du jambon est plus élevé chez l'individu de génotype $n n$. Le cas particulier du gain quotidien sera discuté plus loin. Quant à la contribution du locus $\mathrm{Hal}$ à la variation totale, elle est de $0,9 \%$ pour le gain journalier, de $7,2 \%$ pour la longueur de la carcasse, de $7,7 \%$ pour la proportion de morceaux maigres de $12 \%$ pour la note de conformation finale, de $16 \%$ pour l'indice de compacité 2 , de $11 \%$ pour le pH1 du carré. En revanche, cette contribution est inférieure à $1 \%$ pour les épaisseurs 1 et 2 du gras dorsal, le pH1 du jambon et les deux $\mathrm{pH} 24$.

Concernant l'influence du sexe, celle-ci est significative pour tous les critères, à l'exception du rendement à l'abattage et du $\mathrm{pH} 1$ du carré. On note, chez la 


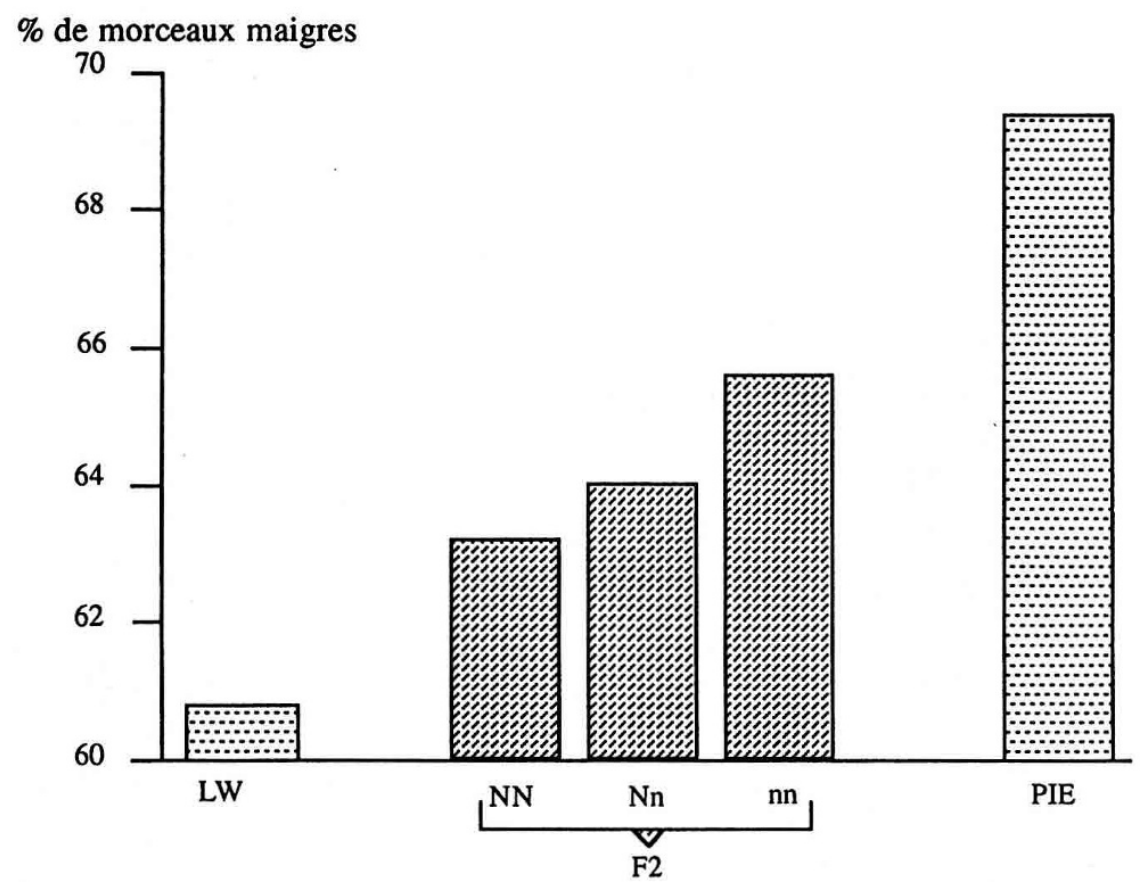

Fig 3. Pourcentage de morceaux maigres des 3 génotypes de la F2 et des races Large White (LW) et Piétrain (PIE).

femelle, une moins bonne croissance pondérale $(-50 \mathrm{gms})$, une carcasse plus longue $(+1 \mathrm{~cm})$ et plus riche en morceaux maigres $(+2,4 \%)$, des notes de conformation plus basses, un indice de compacité 2 supérieur, des mesures du $\mathrm{pH}$ légèrement plus faibles. D'autre part, en ce qui concerne les contributions respectives du locus $\mathrm{Hal}$ et du sexe à la variation totale, on observe une contribution prépondérante du locus pour la longueur de la carcasse, le rendement à l'abattage, le pourcentage d'épaule, les notes de conformation, les indices de compacité, le $\mathrm{pH} 1$ du carré alors que la contribution du sexe l'emporte sur celle du locus pour le gain quotidien, l'épaisseur du gras dorsal et les proportions des différents morceaux, à l'exception de l'épaule. Parmi les différents morceaux, le \% de carré et le \% de bardière sont les plus influencés par le sexe (contribution de $20 \%$ ). On notera que les pourcentages de ces 2 morceaux ne sont pas indépendants.

L'effet du gène (en valeurs absolue et standardisée) ainsi que la déviation de dominance (valeurs absolue et relative) sont données au tableau II. La différence entre les 2 homozygotes est significative pour tous les critères à l'exception du gain quotidien. Un effet génique positif signifie que la valeur métrique de l'homozygote $n n$ est supérieure à celle de l'homozygote $N N$, inversement si l'effet génique est négatif. L'examen des effets géniques standardisés révèle que cet effet approche ou dépasse la valeur d'une déviation standard pour la longueur de la carcasse, les pourcentages de morceaux nobles et de morceaux gras, les notes de conformation, les indices de compacité (spécialement l'indice de compacité 2), le pH1 du carré. 


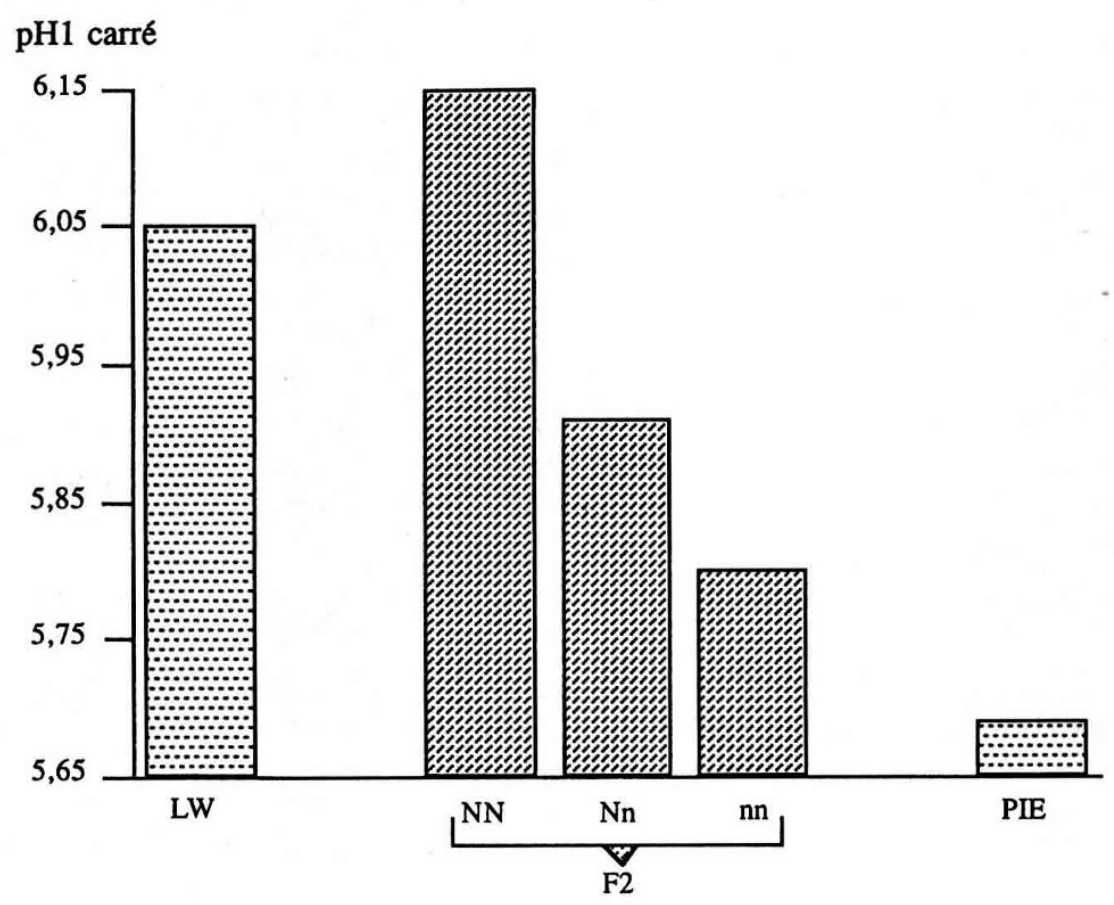

Fig 4. Mesure du pH1 du carré (Longissimus dorsi) des 3 génotypes de la F2 et des races Large White (LW) et Piétrain (PIE).

Un gène est souvent qualifié de «majeur» lorsque son effet dépasse un écart type. L'allèle $n$ exerce donc bien un effet majeur sur les critères énumérés ci-dessus.

L'écart de dominance est significatif pour tous les critères à l'exception de la paroi ventrale, et des $\mathrm{pH} 1$ et $\mathrm{pH} 24 \mathrm{du}$ jambon. Le cas du gain quotidien est particulier dans la mesure où l'hétérozygote est supérieur à chacun des 2 homozygotes. Wittman et al (1993) ont fait la même observation sur des castrats de race DL (Landrace Allemand). L'écart de dominance est positif pour la longueur de la carcasse, l'épaisseur du gras dorsal, les pourcentages de morceaux gras et négatif pour le rendement à l'abattage, pour le pourcentage de morceaux maigres, les notes de conformation et les indices de compacité. Ce fait a également été constaté par Wittman et al (1993) à propos de la longueur de la carcasse et du pourcentage de viande. De plus, alors que pour le rendement à l'abattage l'écart relatif est de $100 \%$, ce qui signifie une récessivité totale de l'allèle $n$, en revanche, cet écart relatif est inférieur à $100 \%$ pour les autres critères, ce qui implique, dans ce cas, une récessivité partielle de l'allèle $n$. En revanche, pour le pH1 du carré, l'allèle $n$ se comporte comme un dominant partiel, puisque l'hétérozygote $N n$ est plus proche de l'homozygote $n n$ que de l'homozygote $N N$.

La F2 se composait de $1 / 4$ de sujets tachetés noirs et de $3 / 4$ de sujets blancs, proportions correspondant à la ségrégation de la paire d'allèles $I$ et $i$, l'allèle $I$ dominant ayant été apporté par le grand-parent Large White et l'allèle $i$ 
Tableau II. Effet du gène et écart de dominance au locus Hal.

\begin{tabular}{|c|c|c|c|c|}
\hline Caractères & $\begin{array}{r}\text { Effet du gène } \\
\text { nn-NN=2a }\end{array}$ & $\begin{array}{c}\text { Effet standardisé } \\
\mathrm{P} \\
\end{array}$ & $\begin{array}{r}\text { Écart de } \\
\text { dominance }\end{array}$ & Écart relatif $(\%) \mathrm{P}$ \\
\hline $\begin{array}{l}\text { Gain quotidien } \\
(\mathrm{kg})\end{array}$ & $\begin{array}{r}0,012 \\
\pm 0,008\end{array}$ & $\begin{array}{l}0,130 \\
+\end{array}$ & $\begin{array}{r}0,018 \\
\pm 0,005\end{array}$ & $\begin{array}{c}300,0 \\
* * *\end{array}$ \\
\hline $\begin{array}{l}\text { Longueur } \\
\quad(\mathrm{cm})\end{array}$ & $\begin{array}{r}-1,895 \\
\pm 0,178\end{array}$ & $\begin{array}{l}-0,856 \\
* * *\end{array}$ & $\begin{array}{r}0,501 \\
\pm 0,128\end{array}$ & $\begin{array}{l}52,91 \\
* * *\end{array}$ \\
\hline $\begin{array}{l}\text { Épaisseur du gras } \\
\text { dorsal }(\mathrm{cou})(\mathrm{cm})\end{array}$ & $\begin{array}{r}-0,161 \\
\pm 0,034\end{array}$ & $\begin{array}{c}-0,293 \\
* * *\end{array}$ & $\begin{array}{r}0,056 \\
\pm 0,032\end{array}$ & $\underset{*}{69,57}$ \\
\hline $\begin{array}{l}\text { Épaisseur du gras } \\
\text { dorsal }\left(1^{\text {re }} \text { vertèbre }\right. \\
\text { lombaire })(\mathrm{cm})\end{array}$ & $\begin{array}{r}-0,080 \\
\pm 0,038\end{array}$ & $\begin{array}{c}-0,168 \\
*\end{array}$ & $\begin{array}{r}0,038 \\
\pm 0,027\end{array}$ & $\begin{array}{l}96,07 \\
\mathrm{NS}\end{array}$ \\
\hline $\begin{array}{l}\text { Épaisseur du gras } \\
\quad \text { dorsal (croupe) }(\mathrm{cm})\end{array}$ & $\begin{array}{r}-0,274 \\
\pm 0,035\end{array}$ & $\begin{array}{c}-0,619 \\
* * *\end{array}$ & $\begin{array}{l}0,095 \\
0,026\end{array}$ & $\begin{array}{l}69,72 \\
* * *\end{array}$ \\
\hline $\begin{array}{l}\text { Épaisseur moyenne } \\
\text { du gras dorsal }(\mathrm{cm})\end{array}$ & $\begin{array}{r}-0,172 \\
\pm 0,030\end{array}$ & $\begin{array}{c}-0,455 \\
* * *\end{array}$ & $\begin{array}{r}0,063 \\
\pm 0,026\end{array}$ & $\begin{array}{l}73,49 \\
* * *\end{array}$ \\
\hline Rendement (\%) & $\begin{array}{r}0,874 \\
\pm 0,140\end{array}$ & $\begin{array}{l}0,503 \\
* * *\end{array}$ & $\begin{array}{r}-0,467 \\
\pm 0,100\end{array}$ & $\begin{array}{c}-106,9 \\
* * *\end{array}$ \\
\hline Carré (\%) & $\begin{array}{r}0,975 \\
\pm 0,105\end{array}$ & $\begin{array}{l}0,744 \\
* * *\end{array}$ & $\begin{array}{r}-0,180 \\
\pm 0,076\end{array}$ & $\begin{array}{c}-36,92 \\
* *\end{array}$ \\
\hline Jambon (\%) & $\begin{array}{r}0,936 \\
\pm 0,089\end{array}$ & $\begin{array}{l}0,847 \\
* * *\end{array}$ & $\begin{array}{r}-0,135 \\
\pm 0,064\end{array}$ & $\begin{array}{c}-28,85 \\
*\end{array}$ \\
\hline Épaule (\%) & $\begin{array}{r}0,461 \\
\pm 0,054\end{array}$ & $\begin{array}{l}0,688 \\
* * *\end{array}$ & $\begin{array}{r}-0,091 \\
\pm 0,039\end{array}$ & $\begin{array}{l}-39,48 \\
* *\end{array}$ \\
\hline $\begin{array}{l}\text { Morceaux } \\
\text { maigres (\%) }\end{array}$ & $\begin{array}{r}2,371 \\
\pm 0,187\end{array}$ & $\begin{array}{l}1,017 \\
* * *\end{array}$ & $\begin{array}{r}-0,406 \\
\pm 0,134\end{array}$ & $\begin{array}{l}-34,25 \\
* * *\end{array}$ \\
\hline Bardière (\%) & $\begin{array}{r}-0,642 \\
\pm 0,102\end{array}$ & $\begin{array}{l}-0,505 \\
* * *\end{array}$ & $\begin{array}{r}0,210 \\
\pm 0,073\end{array}$ & $\begin{array}{l}65,44 \\
* * *\end{array}$ \\
\hline $\begin{array}{l}\text { Partie ventrale } \\
\quad(\%)\end{array}$ & $\begin{array}{r}-0,594 \\
\pm 0,107\end{array}$ & $\begin{array}{c}-0,610 \\
* * *\end{array}$ & $\begin{array}{r}0,024 \\
\pm 0,056\end{array}$ & $\begin{array}{l}8,08 \\
\mathrm{NS}\end{array}$ \\
\hline $\begin{array}{l}\text { Morceaux gras } \\
\quad(\%)\end{array}$ & $\begin{array}{r}-2,184 \\
\pm 0,184\end{array}$ & $\begin{array}{c}-0,958 \\
* * *\end{array}$ & $\begin{array}{r}0,328 \\
+0,132\end{array}$ & $\begin{array}{l}30,07 \\
* *\end{array}$ \\
\hline $\begin{array}{l}\text { Conformation sevrage } \\
(0 \text { à 10) }\end{array}$ & $\begin{array}{r}1,927 \\
\pm 0,173\end{array}$ & $\begin{array}{l}0,913 \\
* * *\end{array}$ & $\begin{array}{r}-0,350 \\
\pm 0,124\end{array}$ & $\begin{array}{l}-36,35 \\
* *\end{array}$ \\
\hline $\begin{array}{l}\text { Conformation finale } \\
(0 \text { à } 10)\end{array}$ & $\begin{array}{r}1,872 \\
\pm 0,152\end{array}$ & $\begin{array}{l}1,091 \\
* * *\end{array}$ & $\begin{array}{r}-0,306 \\
\pm 0,114\end{array}$ & $\begin{array}{l}-32,69 \\
* * *\end{array}$ \\
\hline $\begin{array}{l}\text { Indice } \\
\text { de compacité } 1\end{array}$ & $\begin{array}{r}0,034 \\
\pm 0,003\end{array}$ & $\begin{array}{l}0,783 \\
* * *\end{array}$ & $\begin{array}{r}-0,013 \\
\pm 0,012\end{array}$ & $\begin{array}{l}-76,47 \\
* * *\end{array}$ \\
\hline $\begin{array}{l}\text { Indice } \\
\text { de compacité } 2\end{array}$ & $\begin{array}{r}0,046 \\
\pm 0,003\end{array}$ & $\begin{array}{l}1,405 \\
* * *\end{array}$ & $\begin{array}{r}-0,011 \\
\pm 0,002\end{array}$ & $\begin{array}{c}-47,83 \\
* * *\end{array}$ \\
\hline
\end{tabular}


Tableau II. (suite)

\begin{tabular}{lrcrc}
\hline Caractères & $\begin{array}{r}\text { Effet du gène } \\
\text { nn-NN=2a }\end{array}$ & $\begin{array}{c}\text { Effet standardisé } \\
\mathrm{P}\end{array}$ & $\begin{array}{r}\text { Écart de } \\
\text { dominance }\end{array}$ & Écart relatif (\%) P \\
\hline pH1 carré & $-0,353$ & 1,107 & $-0,064$ & $-36,26$ \\
m Longissimus dorsi & $\pm 0,026$ & $* * *$ & $\pm 0,019$ & $* * *$ \\
pH1 jambon & $-0,074$ & 0,252 & 0,010 & 28,55 \\
m Gracilis & $\pm 0,023$ & $* * *$ & $\pm 0,017$ & NS \\
pH24 carré & $-0,057$ & 0,283 & $-0,021$ & $-73,80$ \\
m Longissimus dorsi & $\pm 0,024$ & $* *$ & $\pm 0,012$ & + \\
pH24 jambon & 0,052 & 0,202 & $-0,015$ & $-59,61$ \\
m Gracilis & $\pm 0,019$ & $* *$ & $\pm 0,015$ & NS \\
\hline
\end{tabular}

NS : $P>0,10 ;+: P<0,10 ;^{*}: P<0,05 ;^{* *}: P<0,01 ;^{* * *}: P<0,001$.

récessif, par le grand-parent Piétrain. Le phénotype blanc recouvre les génotypes $I I$ et $I i$ dans les proportions $1 / 3,2 / 3$ tandis que le phénotype tacheté correspond au seul génotype $i i$. Le phénotype de robe s'est avéré significatif pour certains critères de composition de carcasse comme les pourcentages de morceaux maigres, de morceaux gras, l'indice de compacité 2 (tableau III). On a ainsi observé qu'au sein de cette F2 le phénotype tacheté allait de pair avec une meilleure composition de carcasse. Si on considère le pourcentage de morceaux maigres, la contribution du locus I ( 2 phénotypes) à la variation est de $0,7 \%$ contre $8,5 \%$ pour le locus $\mathrm{Hal}$. Cette observation pourrait signifier la présence, sur le chromosome porteur du locus $I$, de gènes mineurs influençant la composition de la carcasse, les allèles favorables étant apportés par le Piétrain en association avec l'allèle $i$ du tacheté.

Tableau III. Effet du phénotype de robe sur certains caractères. Moyennes des moindres carrés ( \pm erreur standard).

\begin{tabular}{lccc}
\hline Caractères & Robe tachetée & Robe blanche & $P$ \\
\hline Carré (\%) & $24,59 \pm 0,07$ & $24,42 \pm 0,05$ & $*$ \\
Jambon (\%) & $23,35 \pm 0,06$ & $23,06 \pm 0,04$ & $* * *$ \\
Épaule (\%) & $16,56( \pm 0,03)$ & $16,46( \pm 0,02)$ & $*$ \\
Morceaux maigres (\%) & $64,50 \pm 0,12$ & $63,94 \pm 0,08$ & $* * *$ \\
Bardière (\%) & $6,96 \pm 0,07$ & $7,19 \pm 0,05$ & $* * *$ \\
Paroi ventrale (\%) & $15,97 \pm 0,05$ & $16,14 \pm 0,04$ & $* * *$ \\
Morceaux gras (\%) & $28,78 \pm 0,12$ & $29,33 \pm 0,08$ & $* * *$ \\
Indice de compacité 2 & $0,644 \pm 0,002$ & $0,640 \pm 0,001$ & $*$ \\
\hline
\end{tabular}

* $P<0,05 ;{ }^{* *} P<0,01$; *** $P<0,001$.

L'influence du sexe sur le classement commercial des carcasses est bien connue, les carcasses des femelles, plus maigres, obtenant un meilleur classement que celles des castrats, quel que soit par ailleurs le génotype du locus $\mathrm{Hal}$ (tableau IV). L'effet de ce génotype sur le classement est tout aussi évident que l'on envisage les pourcentages de sujets figurant dans les classes E et plus, dans les classes AA et plus ou dans les classes A1 et plus. 
Tableau IV. Influence du génotype au locus $\mathrm{Hal}$ et du sexe sur le classement commercial des carcasses. Répartition en \% dans les différentes classes.

\begin{tabular}{lcccccc}
\hline \multirow{2}{*}{$\begin{array}{l}\text { Classe } \\
\text { commerciale }\end{array}$} & \multicolumn{3}{c}{$\mathrm{NN}$} & \multicolumn{2}{c}{ Génotype halothane } \\
& Castrats & Femelles & Castrats & Femelles & Castrats & Femelles \\
\hline EE & - & - & - & - & - & 1,64 \\
E & - & - & - & 2,61 & 2,04 & 9,29 \\
AA & - & 4,55 & 0,93 & 6,64 & 7,14 & 18,58 \\
A1 & 17,07 & 34,09 & 29,21 & 56,40 & 49,49 & 59,56 \\
A2 & 18,54 & 8,18 & 26,17 & 5,92 & 24,49 & 7,10 \\
A3 & 8,29 & - & 7,94 & 1,18 & 5,10 & 0,55 \\
B1 & 24,88 & 47,27 & 22,90 & 23,70 & 9,69 & 3,28 \\
B2 & 24,39 & 5,45 & 10,51 & 3,32 & 1,05 & - \\
B3 & 6,83 & 0,45 & 2,34 & 0,24 & - & - \\
\hline$n$ & 205 & 220 & 428 & 422 & 196 & 183 \\
\hline E et plus & - & - & - & 2,61 & 2,04 & 10,93 \\
AA et plus & - & 4,55 & 0,93 & 9,25 & 9,18 & 29,51 \\
A1 et plus & 17,07 & 38,64 & 30,14 & 65,65 & 58,67 & 89,07 \\
\hline
\end{tabular}

\section{DISCUSSION ET CONCLUSION}

En l'absence d'un marqueur utile ou d'un test génomique direct, l'étude des effets du locus $\mathrm{Hal}$ sur les performances zootechniques s'est généralement limitée à la comparaison entre sujets halothane positifs et sujets halothane négatifs, c'est-àdire à une comparaison entre phénotypes (Webb, 1981; Monin et al, 1981; Simpson et Webb, 1989). Or, la classe des sujets halothane négatifs peut se composer à la fois d'homozygotes $N N$ et d'hétérozygotes $N$, voire d'homozygotes $n n$, en proportions variables.

L'expérience rapportée ici a été construite de telle manière que les 3 génotypes au locus $\mathrm{Hal}$ puissent être identifiés avec une précision très élevée, ce qui fut confirmé ultérieurement lors de l'introduction du test génomique au locus $R Y R$ 1 du récepteur membranaire à la ryanodine (Grobet et al, 1992; Grobet, 1993). Dès lors, les effets du gène et l'écart de dominance pouvaient être valablement estimés, tout au moins pour un contexte génétique constitué à raison de $50 \%$ par chacune des races Piétrain et Large White, car l'ampleur de ces effets peut varier d'un contexte à l'autre (Webb, 1981; Jensen, 1981).

L'effet génique (2a) atteint un écart type pour le pourcentage de morceaux maigres, la note finale de conformation, l'index de compacité 2 , le $\mathrm{pH} 1$ du carré. Cette énumération résume bien les effets principaux de ce gène. Ils concernent essentiellement la conformation, reflet de l'hypertrophie de muscles insérés sur un squelette plus court, et le $\mathrm{pH} 1$ du carré. D'ailleurs, c'est pour ces mêmes caractères que la participation du locus à la variation totale est la plus importante. Les valeurs trouvées ici pour l'effet génique peuvent être comparées à celles trouvées dans la 
littérature bien que les caractères concernés ne soient pas identiquement les mêmes. Dans la synthèse de Webb (1981), on peut lire que la différence moyenne entre les génotypes $n n$ et $N N$ est de $3,9 \%$ pour le pourcentage de maigre, $1,7 \%$ pour le pourcentage de jambon et de longe, $1,2 \%$ pour le rendement à l'abattage, $-1,1 \mathrm{~cm}$ pour la longueur, $-0,26$ pour le $\mathrm{pH} 45^{\prime}$. Jensen et Barton-Gade (1985) trouvent chez le Landrace Danois que les différences entre homozygotes représentent 1,6, 2,3 et 2,2 écarts types pour le pourcentage de maigre respectivement dans la longe, le jambon et la paroi latérale de la carcasse. La différence de longueur était de $-1,8 \mathrm{~cm}$, celle du $\mathrm{pH} 1 \mathrm{du} \mathrm{m}$ Longissimus dorsi de -0,81. Chez des castrats de race DL (Landrace Allemand), Wittmann et al (1993) observent les différences suivantes : longueur : $-5 \mathrm{~cm}$, pourcentage de viande : $+2,7 \%$, pH1 de la côtelette : $-0,79, \mathrm{pH} 1 \mathrm{du}$ jambon : $-0,53$.

D'autre part, si l'allèle $n$ est récessif et à pénétrance incomplète (toutefois supérieure à $90 \%$, Grobet et $a l, 1992$ ), il s'exprime généralement à l'état hétérozygote même si le degré d'expression n'atteint généralement pas la moyenne des 2 homozygotes. Qu'il s'agisse du pourcentage de maigre dans la carcasse ou du $\mathrm{pH} 1$, Jensen et Barton-Gade (1985) et Wittman et al (1993) constatent que l'hétérozygote $N n$ est plus proche de l'homozygote $N N$ que de l'homozygote $n n$. Néanmoins, pour des caractères tels que les notes de conformation, les indices de compacité, le classement commercial des carcasses, cette expression chez l'hétérozygote est suffisante pour expliquer l'avantage sélectif dont le gène à l'état hétérozygote a pu bénéficier dans le cadre d'une sélection basée sur la conformation de l'animal vif ou abattu. Cet avantage sélectif conduisit en fin de compte à la fixation du gène, telle qu'elle s'observe chez le porc Piétrain.

D'autre part, il est évident qu'un porc F2 de génotype $n n$ ne peut être confondu avec un porc Piétrain. En effet, celui-ci a un pourcentage de morceaux maigres de l'ordre de $69,5 \%$ contre $65,5 \%$ chez les sujets $n n$ de la F2 (fig 3) et l'indice de compacité 2 a, en moyenne, la valeur de 0,75 chez le Piétrain pur contre 0,67 chez les sujets $n n$ de la F2. En outre, pour le classement commercial, si chez le porc Piétrain, $70 \%$ des femelles se situent dans les classes $\mathrm{E}+\mathrm{EE}$, en revanche, chez les femelles $n n$ de la F2, ce pourcentage n'est que de $11 \%$. Dès lors, si le génotype $n n$ est nécessaire pour faire un porc Piétrain, cette condition n'est pas suffisante. D'autres gènes dont l'action globale sur la composition de la carcasse est analogue à celle de l'allèle $n$ participent donc à cette différence.

\section{REMERCIEMENTS}

Les auteurs remercient le Dr Christian Schirvel et M Albert Drisket, technicien, qui ont mis en place le troupeau expérimental; la station de recherches porcines de l'INRA à SaintGilles-L'Hermitage (France) pour sa précieuse collaboration dans le choix des animaux fondateurs de race Large White; M Marcel Bustin pour sa gestion de l'élevage; le Dr $S$ Dufrasne du centre d'insémination artificielle d'Argenteau; et $\mathrm{M}^{\text {lle }}$ Nadine Brunetta pour la dactylographie du manuscrit.

Les auteurs remercient également les 2 lecteurs anonymes pour leurs remarques judicieuses.

Ce travail a été réalisé sous les auspices de l'Institut pour l'encouragement de la recherche scientifique dans l'industrie et l'agriculture (IRSIA). 


\section{RÉFÉRENCES}

Eikelenboom G, Minkema D, Van Eldik P, Sybesma W (1978) Production characteristics of Dutch Landrace and Dutch Yorkshire pigs as related to their susceptibility for the halothane-induced malignant hyperthermia syndrome. Livestock Prod Sci 5, 277-284

Grobet L (1993) Diagnostics génomiques. Thèse, université de Liège

Grobet L, Hanset R, Dasnois C (1992) Réponse au test à l'halothane et génotype au locus $R Y R 1$ du récepteur à la ryanodine chez des porcs croisés Piétrain. Ann Méd Vét 136, 249-257

Hanset R, Van Snick G (1972) Les paramètres génétiques des caractères d'engraissement et de carcasse chez le porc Piétrain. Ann Génét Sél Anim 4, 451-467

Jensen P (1981) Carcass and meat quality of pigs with known genotypes for halothane susceptibility. In : Porcine stress and meat quality (T Frøstein, E Slinde, N Standal, eds), Agric Food Res Soc, Ås, Norway, 267-273

Jensen P, Barton-Gade PA (1985) Performance characteristics of pigs with known genotypes for halothane susceptibility. In : Stress susceptibility and meat quality in pigs (JB Ludvigsen, ed), EAAP Publication $n^{\circ} 33,80-87$

Jørgensen PF, Hyldgaard-Jensen J, Eikelenboom J, Moustgaard J (1976) Phosphohexose isomerase (PHI) and porcine halothane sensitivity. Acta Vet Scand 17, 370-372

Kintaba KN, Hanset R, Michaux C (1981) Composition de la carcasse et classement commercial chez le porc de Piétrain et le porc Landrace Belge. Ann Méd Vét 125, 215-228

Lampo P (1981) La sensibilité au stress chez le Landrace Belge. La relation entre le test d'anesthésie à l'halothane, les caractéristiques d'engraissement et de la carcasse. Rev Agric 34, 213-220

Monin G, Sellier P, Ollivier L, Goutefongea R, Girard JP (1980) Carcass characteristics and meat quality of halothane-negative and halothane-positive Piétrain pigs. Meat Sci $5,413-423$

Ollivier L, Sellier P, Monin G (1975) Déterminisme génétique du syndrome d'hyperthermie maligne chez le porc de Piétrain. Ann Gén Sél Anim 7, 159-166

SAS (1985) User's guide: statistics. SAS Inst Inc, Cary, NC, USA (5th ed)

Simpson SP, Webb AJ (1989) Growth and carcass performance of British Landrace pigs heterozygous at the halothane locus. Anim Prod 49, 503-509

Webb AJ (1981) The halothane sensitivity test. In : Porcine stress and meat quality (T Frøstein, E Slinde, N Standal, eds), Agric Food Res Soc, Ås, Norway, 105-124

Webb AJ, Jordan CHC (1978) Halothane sensitivity as a field test for stress-susceptibility in the pig. Anim Prod 26, 157-168

Widar J, Ansay M, Hanset R (1975) Allozymic variation as an estimate of heterozygosity in Belgian pig breeds. Anim Blood Groups Biochem Genet 6, 221-234

Wittmann W, Peschke W, Littmann E, Behringer J, Birkenmaier ST, Dovc P, Förster M (1993) Mast- und Schlachtleistungen von DL-kastraten in Abhängigkeit von MHSgenotyp. Züchtgskde 65, 197-205 